\title{
A pairwise discriminant approach using artificial neural networks for continuous speech recognition
}

\author{
Jun-ichi Takami,* Atsuhiko Kai,** and Shigeki Sagayama* \\ *ATR Interpreting Telephony Research Laboratories, \\ 2-2 Hikaridai, Seika-cho, Soraku-gun, Kyoto, 619-02 Japan \\ **Department of Information and Computer Sciences, \\ Toyohashi University of Technology, \\ 1-1 Hibarigaoka, Tenpaku-cho, Toyohashi, 441 Japan
}

(Received 21 February 1992)

\begin{abstract}
This paper describes a pairwise discrimination approach using artificial neural networks for robust phoneme recognition and its application to continuous speech recognition. Until now, it is known that classification-type neural networks show poor robustness against the difference in speaking rates between training data and testing data. To improve the robustness, we developed Pairwise Discriminant Time-Delay Neural Networks (PD-TDNNs) by applying the principle of pair discrimination to a conventional Time-Delay Neural Network. In this approach, pair discrimination scores for all combinations of two phonemes are calculated by PD-TDNNs, each of which has a less sharp discrimination boundary, and final phoneme candidates are decided by majority decision of the pair discrimination scores. Through phoneme and continuous speech recognition experiments, it was found that this approach performs better than the conventional TDNN.
\end{abstract}

Keywords: Pairwise discriminant approach, Time-Delay Neural Network, Phoneme recognition, Continuous speech recognition, Predictive LR-parser

PACS number: $43.72 . \mathrm{Ne}$

\section{INTRODUCTION}

In recent years, the investigation of speech recognition methods using artificial neural networks ${ }^{1)}$ has received considerable attention. It is well known that classification-type neural networks (i.e. neural networks whose desired output values are either 0 or 1), such as Time-Delay Neural Networks (TDNNs), ${ }^{2)}$ have high performance for phoneme discrimination. However, in the case of continuous speech recognition using a predictive LR-parser, when such neural networks are trained with phoneme data extracted from isolated word utterances, the difference in speaking rates between training data and testing data degrades the continuous speech recognition performance. ${ }^{3 \text { ) }}$

This is due to a lack of robustness to variations in speaking rate. Since the LR-parser treats the phoneme verification scores as a kind of likelihood measure, the phoneme veriners should ideally produce some measure of phoneme similarity. However, although the input data includes acoustic ambiguities, neural network phoneme verifiers tend to classify it into one phoneme category with little ambiguity. This is due to the very sharp discrimination boundaries formed between each phoneme category. In this instance, phoneme verification scores tend to 1 for the first candidate, whether it is correct or not, and 0 for the other candidates.

To overcome these problems, we believe that the following two solutions are effective:

- discriminate each phoneme category using less sharp discrimination boundaries.

- discriminate each phoneme category by a majority 
decision using multiple discrimination boundaries.

To realize these two solutions simultaneously, we developed Pairwise Discriminant Time-Delay Neural Networks (PD-TDNNs), ${ }^{4,5}$ ) based upon the principle of pairwise discrimination. ${ }^{6,7)}$ The significant feature of PD-TDNNs is that the aforementioned two solutions can be achieved simultaneously by a suitable training algorithm, and the principle of majority decision.

In this paper, we describe both a phoneme recognition method using PD-TDNNs and a continuous speech recognition method using phoneme verifiers consisting of the PD-TDNNs. Moreover, we show several experimental results for both Japanese consonant recognition and Japanese phrase recognition, and discuss the advantage of the pairwise discriminant approach.

\section{PAIRWISE DISCRIMINANT TIME-DELAY NEURAL NETWORKS}

\subsection{Architecture of A PD-TDNN}

Figure 1 shows the architecture of a PD-TDNN. A PD-TDNN is a kind of TDNN consisting of four layers to discriminate between two phoneme categories. The input layer consists of 112 units corresponding to input data ( 7 frames of 16 mel-scaled spectrum) and the output layer consists of only 1 unit for an output lying between 1 and 0 .

A PD-TDNN for discrimination between two phoneme categories $p_{i}$ and $p_{j}$, Net $\left(p_{i} / p_{j}\right)$, is trained using the following algorithm: if the input data belongs to category $p_{i}$, category $p_{j}$, or neither category, 1,0 , or 0.5 are given as the respective desired

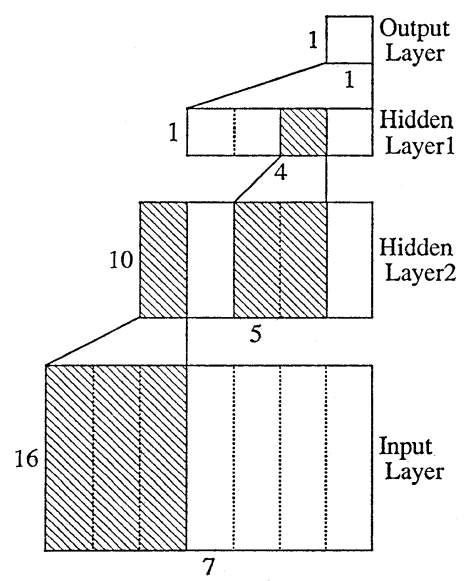

Fig. 1 Architecture of a PD-TDNN. outputs. In this case, the output value of Net $\left(p_{i} / p_{j}\right)$ and the value calculated by subtracting the output value from 1 can be regarded as the pair discrimination scores for categories $p_{i}$ and $p_{j}$, respectively.

In connection with this training algorithm, we improved the training efficiency by introducing a new non-linear function. If a PD-TDNN is trained using a common sigmoid function in the output unit, it is difficult to decrease the error for the data which should output the value 0.5 , because the common sigmoid function has a maximum differential coefficient at the point corresponding to the output value of 0.5 . Accordingly, the non-linear function shown in Eq. (1) is used in the output unit.

$$
f(x)= \begin{cases}\frac{g(x+\alpha)}{2 g(\alpha)} & \text { if }(x<0), \\ 1-\frac{g(-x+\alpha)}{2 g(\alpha)} & \text { if }(x \geq 0),\end{cases}
$$

where $g$ is: $g(x)=\frac{1}{1+e^{-x}}$.

In this equation, $\alpha$ is a value to control the differential coefficient around the point corresponding to the output value of 0.5 . We used $\alpha=3.0$ for the results presented in this paper. Figure 2 shows the form of this function.

\subsection{Phoneme Recognition Method}

Since $N$ categories produce ${ }_{N} \mathrm{C}_{2}$ pairs, ${ }_{N} \mathrm{C}_{2} \mathrm{PD}$ TDNNs are necessary for a phoneme recognition problem having $N$ categories.

First, two pair discrimination scores are calculated from the output value of one PD-TDNN. Respective pair discrimination scores of $p_{i}$ and $p_{j}$ are calculated as follows:

$$
\left(\begin{array}{l}
S\left(p_{i} \mid p_{i}: p_{j}\right)=\operatorname{Out}\left(p_{i} / p_{j}\right) \\
S\left(p_{j} \mid p_{i}: p_{j}\right)=1-\operatorname{Out}\left(p_{i} / p_{j}\right)
\end{array}\right.
$$

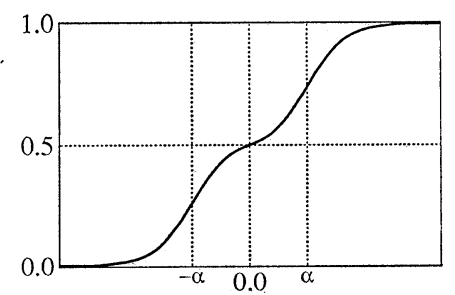

Fig. 2 Non-linear function in the output unit of the PD-TDNN. 


\section{J. TAKAMI et al.: PAIRWISE DISCRIMINANT NEURAL NETWORKS}

In this equation, $S\left(p_{i} \mid p_{i}: p_{j}\right)$ represents the pair discrimination score for category $p_{i}, \operatorname{Out}\left(p_{i} / p_{j}\right)$ represents the output value of $\operatorname{Net}\left(p_{i} / p_{j}\right)$. At this point, there are $N-1$ pair discrimination scores per category.

Second, total scores are calculated by averaging the corresponding $N-1$ pair discrimination scores category by category. A total score for category $p_{i}, T\left(p_{i}\right)$, is obtained by Eq. (3).

$$
T\left(p_{i}\right)=\frac{1}{N-1} \sum_{j \neq i} S\left(p_{i} \mid p_{i}: p_{j}\right) .
$$

Finally, multiple phoneme candidates are selected in descending order of these total scores.

In this method, phoneme candidates are decided by majority decision of all PD-TDNNs, each of which has a less sharp discriminating boundary between the corresponding phoneme pair. Accordingly, even if the wrong outputs are obtained from some PD-TDNNs, the total score $T\left(p_{i}\right)$ is smoothed by averaging the outputs of the individual PDTDNNs. Because of this smoothing, improvement of the recognition performance can be expected.

\section{PHONEME RECOGNITION EXPERIMENTS}

\subsection{Recognition Task}

Phoneme recognition experiments were performed on the task of 6 Japanese consonants, $/ \mathrm{b} /, / \mathrm{d} /, / \mathrm{g} /$, $/ \mathrm{m} /, / \mathrm{n} /, / \mathrm{N} /$, which are easily confused with each other.

\subsection{Utterances and Analysis}

As input data for each network, we used 7 frames of 16-channel mel-scaled spectral coefficients calculated from the phoneme data in the Japanese large vocabulary database uttered by a native male speaker. ${ }^{8)}$ All utterances in this database were digitized at $12 \mathrm{kHz}$. The input data were calculated by the following process: a speech utterance was Hamming windowed and a 256-point FFT computed every $5 \mathrm{~ms}$, 16-channel mel-scaled spectrum coefficients were computed from the power spectrum, adjacent coefficients in time were averaged resulting in an overall $10 \mathrm{~ms}$ frame rate and these coefficients ( 7 frames of 16-channel mel-scaled spectrum coefficients) were normalized to lie between -1 and +1 , with the average at 0 . For both training and testing, every end point of the hand-segmented data was aligned at the center frame of the input layer.

\subsection{Training Data and Testing Data}

For network training and testing, the data extracted from the following utterances uttered by a native male speaker were used:

[Training]

- Even-numbered isolated words of the 5,240 common Japanese words. (5.68 mora/s)

[Testing]

- Odd-numbered isolated words of the 5,240 common Japanese word. ("word": 5.68 mora/s)

- Conversational sentences from a task called "The International Conference Secretarial Service" uttered phrase by phrase. ("phrase": 7.14 mora/s)

- Conversational sentences from a task called "The International Conference Secretarial Service" uttered continuously. ("continuous": 9.56 mora/s)

\subsection{Network Training}

For each Net $\left(p_{i} / p_{j}\right)$ training, 3,000 training data (500 training data for each of 6 categories) were used.

In this cases, the desired outputs for $\operatorname{Net}\left(p_{i} / p_{j}\right)$ were given as 1 for category $p_{i}, 0$ for category $p_{j}$ and 0.5 for the other categories.

During the training, the fast back-propagation learning method $^{9)}$ was used.

\subsection{Comparative Experiment 1 (Use of Basic PD- TDNNs)}

To prove the effect of the training method using the desired output value of 0.5 , we trained Basic PD-TDNNs, each of which has the same architecture as shown in Fig. 1, only with training data for categories $p_{i}$ and $p_{j}$, and tested these networks on the 6-consonant recognition experiments. In this case, 1,000 samples (500 samples for each of corresponding categories $p_{i}$ and $p_{j}$ ) were used for training each $\operatorname{Net}\left(p_{i} / p_{j}\right)$.

In addition, the common sigmoid functions were used in the output units of Basic PD-TDNNs instead of the non-linear function shown in Eq. (1).

\subsection{Comparative Experiment 2 (Use of Conven- tional TDNN)}

To compare the phoneme recognition performances of our method with that of a conventional method, we also performed the same experiments using a conventional TDNN. Figure 3 shows the 


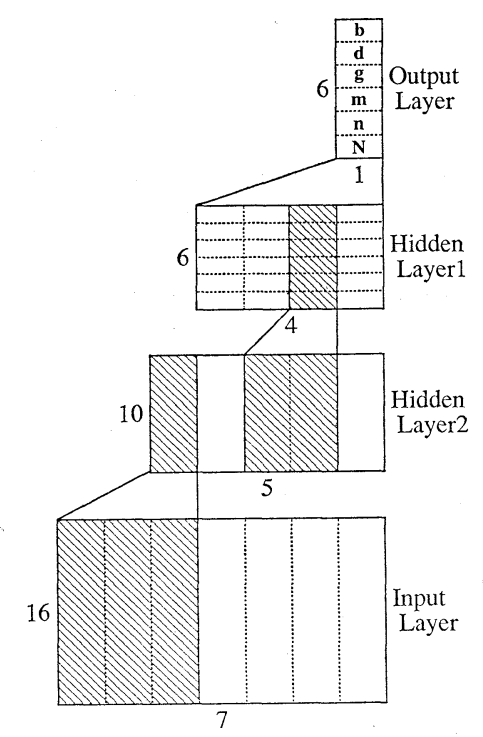

Fig. 3 Architecture of a conventional TDNN.

Table 1 6-consonant recognition experimental results.

\begin{tabular}{lcll}
\hline \multicolumn{4}{c}{ Recognition rates for top 1 (top 3) choice(s) (\%) } \\
\hline Testing data & PD-TDNNs & Basic & TDNN \\
& & PD-TDNNs & \\
"word" & $97.3(99.8)$ & $94.7(99.7)$ & $95.2(99.8)$ \\
"phrase" & $86.2(98.1)$ & $77.2(97.0)$ & $84.5(95.3)$ \\
"continuous" & $81.6(96.7)$ & $73.2(96.3)$ & $77.8(92.2)$ \\
\hline
\end{tabular}

architecture of the TDNN. This network was trained with the same 3,000 training data as used for each PD-TDNN training using the conventional training method, i.e., the desired output of 1 was given for the output unit corresponding to the correct phoneme category, and that of 0 was given for the other output units.

\subsection{Pheoneme Recognition Experimental Results}

Table 1 shows the 6-consonant recognition experimental results obtained using PD-TDNNs, Basic PD-TDNNs, and TDNN, respectively.

From these results, it was found that, though the recognition rates obtained using Basic PD-TDNN are lower than those obtained using a conventional TDNN for the top 1 choice, the Basic PD-TDNNs show higher recognition performances than the
TDNN for the top 3 choices with the exception of "word" utterances. Moreover, it was found that the method using PD-TDNNs gives higher recognition rates for both the top 1 choice and the top 3 choices for all testing data than either of the other two methods.

\section{DISCUSSION ON THE BASIC PERFORMANCE OF THE PAIRWISE DISCRIMINANT APPROACH}

It is well known that classification-type neural networks, like a conventional TDNN, tend to output the value near 1 for the first candidate, whether it is correct or not, and 0 for the other candidates. So when the first candidate is wrong, the output value for the true category become almost 0 . This behavior is often connected with unrecoverable errors.

On the other hand, the method using PD-TDNNs can reflect the likelihood of the selected candidates on its output values. This effect is caused from two significant features, i.e., use of less sharp discrimination boundaries and use of multiple discrimination boundaries.

To support these claims, we investigate differences of the output values among methods using the aforementioned three types of neural networks from the two following points of view:

\subsection{Control Effect of Discrimination Boundaries}

One significant feature of the PD-TDNNs is that each network is trained not only with the desired output values of 1 and 0 but also with the value 0.5 . This training algorithm suppresses the formation of sharp discrimination boundaries since sharp discrimination boundaries are hard to concentrate output values for various input data of multiple categories near 0.5 .

To prove such an effect, we observed which areas of the non-linear function were used at the output unit to generate the output values for each category. Figures 4(a), 4(b) and 4(c) show the respective histograms of 0.1 steps calculated using the training data.

From these figures, it was found that a conventional TDNN represents each output value, 1 and 0 , by repeated use of the saturated areas on the sigmoid function. This means that the TDNN has sharp discrimination boundaries among categories. On the other hand, it was found that each PDTDNN represents the respective output values, 1,0 , 


\section{J. TAKAMI et al.: PAIRWISE DISCRIMINANT NEURAL NETWORKS}

and 0.5 , using non-saturated areas of the non-linear output function. Moreover, it was found that, though Basic PD-TDNNs have not so sharp dis-

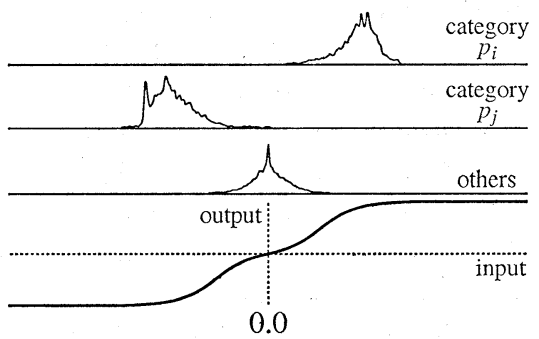

(a) PD-TDNNs

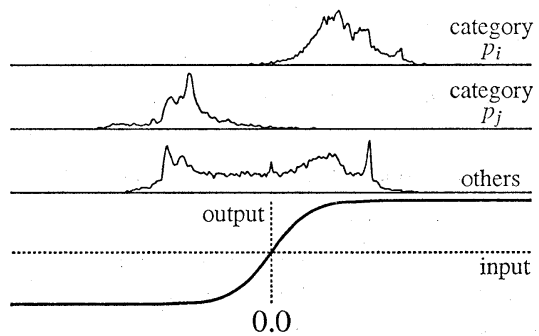

(b) Basic PD-TDNNs

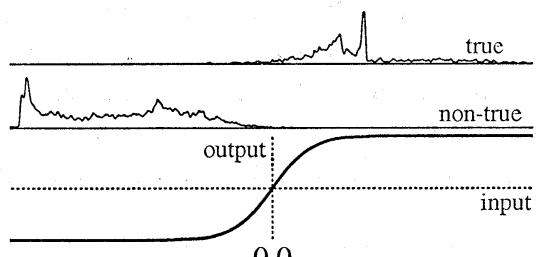

(c) TDNN

Fig. 4 Histograms by category of input values at the output unit before applying the non-linear function (below). crimination boundaries, the histogram for categories of neither $p_{i}$ nor $p_{j}$ distributes wider than that of PD-TDNNs because the Basic PD-TDNNs are not trained such categories with the desired output value of 0.5 .

From these results, it was found that the training using the middle value of 0.5 is effective to form less sharp discrimination boundaries.

\subsection{Smoothing Effect of Network Output Values}

To construct continuous speech recognition systems by combining phoneme recognizers with natural language processors, robust phoneme recognition is important. Nevertheless, the conventional TDNN does not have sufficient robustness so non-training data easily cause unrecoverable errors. On the other hand, PD-TDNNs are expected to have robust phoneme recognition performances, and to reduce such unrecoverable errors.

To support this claim, we observed the recognition scores using scatter plots of the score for true category $v s$. the maximum score for non-true categories. Figures 5(a), 5(b) and 5(c) show the respective scatter plots calculated using the "continuous" testing data. In each figure, the horizontal axis shows the recognition score for the true phoneme category and the vertical axis shows the maximum recognition score for the non-true phoneme categories. In these figures, the data located below the dotted line have been recognized correctly as the first candidates.

From these figures, it was found that almost all the points obtained from the conventional TDNN are scattered around the side of the square including many errors, each of which has a very small recogni-

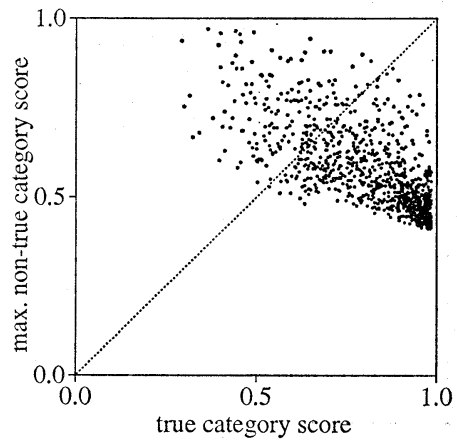

(a) PD-TDNNs

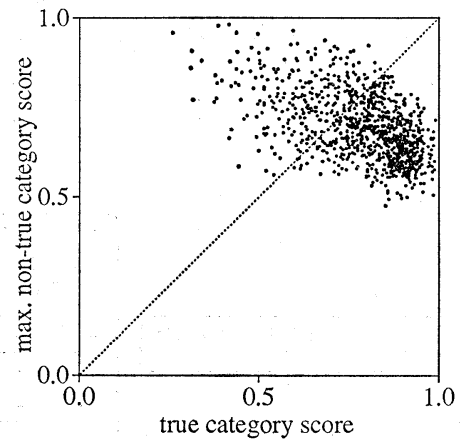

(b) Basic PD-TDNNs

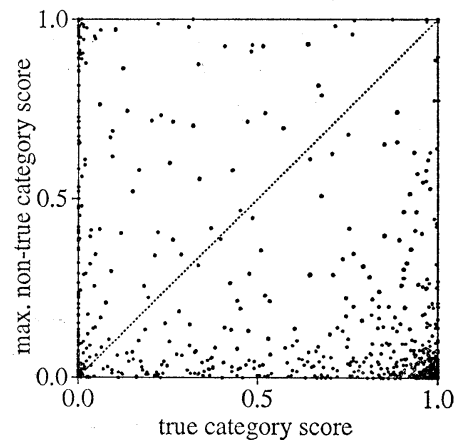

(c) TDNN

Fig. 5 Scatter plot of score for true category $v s$. maximum score for non-true categories. 
tion score for the true phoneme category (i.e. unrecoverable errors). On the other hand, almost all points obtained from both the PD-TDNNs and the Basic PD-TDNNs are distributed in the middle of the square. Moreover, it was found that the distribution of each point obtained from the PDTDNNs is located lower in the square than that obtained from the Basic PD-TDNNs.

From these results, we found the effectiveness of majority decision using less sharp discrimination boundaries.

\section{CONTINUOUS SPEECH RECOGNITION EXPERIMENTS}

\subsection{Combining PD-TDNNs and Predictive LR-} Parser

LR-parsers are well known in the field of programming languages, and can be applied to a large class of context-free grammars. The generalized LR-parser ${ }^{10)}$ is a kind of LR-parser, and has been extended to handle arbitrary context-free grammars. The predictive LR-parser for continuous speech recognition is based on the generalized LR-parser and can predict the next phonemes in input speech based on the currently processed phonemes. Until now, an HMM-LR method, ${ }^{11)}$ which uses an HMM phoneme recognition method as phoneme verifiers, and a TDNN-LR method, ${ }^{12)}$ which uses the conventional TDNN phoneme recognition method as a phoneme verifier, were proposed. We tried to achieve accurate continuous speech recognition by combining phoneme verifiers consisting of PDTDNNs, with the predictive LR-parser. ${ }^{13)}$ The block diagram of this system is shown in Fig. 6.

\subsection{Network Architecture and Training Samples}

For these experiments, since 25 categories consisting of 24 phonemes of $/ \mathrm{b} /, / \mathrm{d} /, / \mathrm{g} /, / \mathrm{p} /, / \mathrm{t} /, / \mathrm{k} /$, $/ \mathrm{m} /, / \mathrm{n} /, / \mathrm{N} /, / \mathrm{s} /, / \mathrm{sh} /, / \mathrm{h} /, / \mathrm{z} /, / \mathrm{ch} /, / \mathrm{ts} /, / \mathrm{zh} /, / \mathrm{r} /$, $/ \mathrm{w} /, / \mathrm{y} /, / \mathrm{a} /, / \mathrm{i} /, / \mathrm{u} /, / \mathrm{e} /, / \mathrm{o} /$ and a silence were used, $300\left(={ }_{25} \mathrm{C}_{2}\right)$ PD-TDNNs were formed. Each PDTDNN has the same architecture shown in Fig. 1 except that the first hidden layer consists of $16 \times 5$ units rather than $10 \times 5$ units.

All PD-TDNNs were formed by training with training data extracted from even-numbered isolated word utterances. At this time, training data was extracted from not only one point of each phoneme period but also multiple points of the phoneme period. Extraction was performed with a $10 \mathrm{~ms}$ frame shift over the phoneme period because the phoneme verifiers should produce correct phoneme verification scores for the entire phoneme period. For each Net $\left(p_{i} / p_{j}\right)$ training, 6,900 training data (2,300 training data for each of the corresponding categories $p_{i}$ and $p_{j}$, and 100 data for each of the remaining 23 categories) were used.

\subsection{Comparative Experiment (Use of Conven- tional TDNN)}

We also performed recognition experiments using a phoneme verifier consisting of a conventional TDNN for comparison. The architecture of this network, which is similar to that shown in Fig. 3, consists of an input layer having $16 \times 7$ units, a first hidden layer having $250 \times 5$ units, a second hidden layer having $25 \times 4$ units and an output layer having 25 units. For training this network, 50,000 data (2,000 data for each of 25 categories) were used.

\subsection{Conversion of Phoneme Verification Scores}

As shown in Fig. 5(a), the range of phoneme verification scores obtained by the PD-TDNNs is, on average, narrower than that obtained by the conventional TDNN. This is because the expectation of each phoneme verification score, $T\left(p_{i}\right)$, lies not between 0 and 1 but between $(N-2) / 2(N-1)(N$ is the number of categories) and 1. Hence, in this system, each phoneme verification score calculated by the PD-TDNNs, $T\left(p_{i}\right)$, is used after being con-

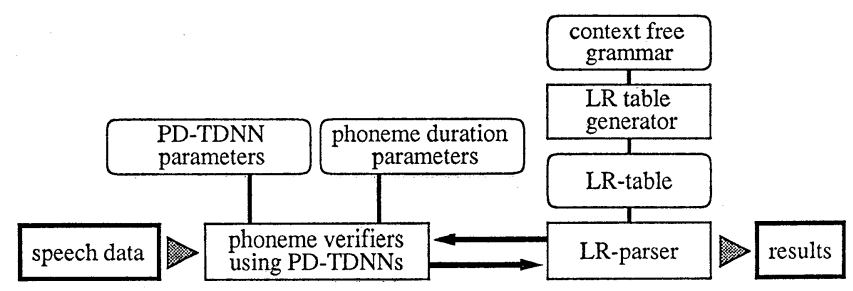

Fig. 6 Block diagram of the continuous speech recognition system. 


\section{J. TAKAMI et al.: PAIRWISE DISCRIMINANT NEURAL NETWORKS}

Table 2 Complexity of grammars.

\begin{tabular}{lrr}
\hline \multicolumn{1}{c}{ Grammar } & "small" & "large" \\
\hline Number of words & 275 & 1,035 \\
Number of rules & 607 & 1,672 \\
Number of LR-states & 1,342 & 4,867 \\
Phoneme perplexity & 3.9 & 5.9 \\
\hline
\end{tabular}

Table 3 Phrase recognition experimental results.

\begin{tabular}{|c|c|c|}
\hline \multicolumn{3}{|c|}{ Recognition rates for top 1 (top 5) choice(s) $(\%)$} \\
\hline Grammar & PD-TDNNs & TDNN \\
\hline "small" & $82.4 \quad(97.5)$ & $71.2 \quad(95.0)$ \\
\hline "large" & $75.5 \quad(92.5)$ & $68.0 \quad(88.1)$ \\
\hline
\end{tabular}

verted to $T_{\text {conv }}\left(p_{i}\right)$ using Eq. (4) to expand the range.

$$
\begin{aligned}
& T_{\text {conv }}\left(p_{i}\right)=\frac{T\left(p_{i}\right)-\beta}{1-\beta}(1-\gamma)+\gamma, \\
& \text { where } \beta \text { is: } \quad \beta=\frac{N-2}{2(N-1)} .
\end{aligned}
$$

In this equation, $\gamma$ is a flooring constant to prevent the phoneme verification scores from becoming too small. We used $\gamma=0.1$ in this paper.

\subsection{Phrase Recognition Experimental Results}

We tested this method on Japanese phrase recognition using both a "small" grammar and a "large" grammar. The complexity of these grammars are shown in Table 2. Table 3 shows the experimental results.

With the "small" grammar and the "large" grammar, we obtained phrase recognition rates of $82.4 \%$ or $75.5 \%$, respectively, for the top 1 choice $(11.2 \%$ or $7.5 \%$ are the respective improvements compared with the method using the conventional TDNN). In addition, recognition rates for the top 5 choices obtained using the PD-TDNNs are also higher than those obtained using the conventional TDNN. From these results, it was found that the phoneme recognition method using PD-TDNNs has high performance not only for phoneme recognition but also for continuous speech recognition.

\section{CONCLUSIONS}

In this paper, we described a phoneme recogni- tion method using Pairwise Discriminant TimeDelay Neural Networks (PD-TDNNs) and a continuous speech recognition method using the PDTDNNs as phoneme verifiers. Through both Japanese 6-consonant recognition experiments and Japanese phrase speech recognition experiments, we proved that the pairwise discriminant approach using artificial neural networks has high recognition performance for various speaking rates. In addition, we investigated the effectiveness of the pairwise discriminant approach, and showed its advantage.

\section{ACKNOWLEDGMENTS}

We would like to thank Dr. A. Kurematsu, President, ATR Interpreting Telephony Research Laboratories, for his continuous support of this work. We also acknowledge Dr. H. Sawai and Mr. Y. Komori for their help in carrying out the experiments and the other members of the Speech Processing Department for their discussion and encouragement.

\section{REFERENCES}

1) D. E. Rumelhart and J. L. McClelland, Parallel Distributed Processing; Explorations in the Micro Structure of Cognition (MIT Press, Cambridge MA, 1986).

2) A. Waibel, T. Hanazawa, G. Hinton, K. Shikano, and K. Lang, "Phoneme recognition using timedelay neural networks," IEEE Trans. Acoust. Speech Signal Process. ASSP-37, 328-339 (1989).

3) Y. Minami, T. Hanazawa, H. Iwamida, E. McDermott, K. Shikano, and M. Nakagawa, "On sensitivity and robustness of HMM and neural network speech recognition algorithms," Proc. ICSLP 90, Vol. 2, 1345-1348 (1990).

4) J. Takami and S. Sagayama, "Phoneme recognition by pairwise discriminant TDNNs," Proc. ICSLP 90, Vol. 1, 677-680 (1990).

5) J. Takami and S. Sagayama, "A pairwise discriminant approach to robust phoneme recognition by time-delay neural networks," Proc. ICASSP-91, Vol. 1, 89-92 (1991).

6) A. Amano, T. Aritsuka, N. Hataoka, and A. Ichikawa, "On the use of neural networks and fuzzy logic in speech recognition," Proc. IJCNN 89, Vol. 1, 301-305 (1989).

7) S. Kitazawa, H. Kojima, and S. Doshita, "Multiclass pattern recognition based on pairwise discrimination," Trans. IEICE J72-A, 41-48 (1989) (in Japanese).

8) K. Takeda, Y. Sagisaka, and S. Katagiri, "A Japanese speech database for various kinds of research 
purpose," J. Acoust. Soc. Jpn. (J) 44, 747-754 (1988) (in Japanese).

9) P. Haffner, A. Waibel, and K. Shikano, "Fast backpropagation learning methods for neural networks in speech," Proc. Autumn Meet. Acoust. Soc. Jpn., Vol. 1, 203-204 (1988).

10) M. Tomita, Efficient Parsing for Natural LanguageA Fast Algorithm for Practical Systems (Kluwer Academic Publishers, Boston, 1986).

11) K. Kita, T. Kawabata, and H. Shikano, "HMM continuous speech recognition using predictive LR parsing," Proc. ICASSP-89, Vol. 2, 703-706 (1989).

12) H. Sawai, "The TDNN-LR large-vocabulary and continuous speech recognition system," Proc. ICSLP 90, Vol. 2, 1349-1352 (1990).

13) J. Takami, A. Kai, and S. Sagayama, "Speech recognition by combining pairwise discriminant time-delay neural networks and predictive LRparser," Proc. NNSP 91, 327-336 (1991).

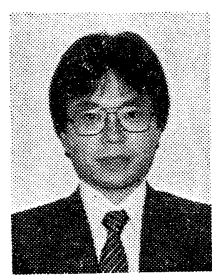

Jun-ichi Takami was born in Tokyo, Japan on April 2, 1961. He received the B. E. degree in Acoustic Engineering from Kyushu Institute of Design, Japan, in 1984. From 1984 to 1989, he worked at the Central Research \& Development Center, Victor Company of Japan Ltd., where he was engaged in digital signal processing research. Since 1989, he has been with the ATR Interpreting Telephony Research Laboratories, Kyoto, Japan, where he is currently a Researcher in the Speech Proecssing Department.

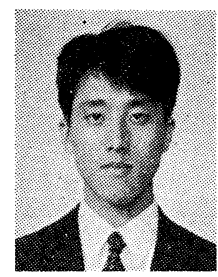

Atsuhiko Kai was born in Kumamoto, Japan, in 1968. He received the B. E. degree in Information and Computer Sciences from Toyohashi University of Technology, Toyohashi, in 1991. $\mathrm{He}$ is currently working toward the M. Eng. He has been engaged in research on a speech recognition and understanding system.

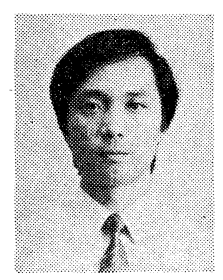

Shigeki Sagayama was born in Hyogo Prefecture, Japan, in 1948. He received $\mathrm{BE}$ and $\mathrm{ME}$ degrees from University of Tokyo, Tokyo, Japan, in 1972 and 1974, respectively, both in Mathematical Engineering and Instrumentation Physics. He joined Electrical Communications Laboratories, Nippon Telegraph and Telephone Public Corporation, in 1974. Since 1990, he has been with ATR Interpreting Telephony Research Laboratories, where he is currently in charge of Head, Speech Processing Department. His research interests include speech recognition, speech synthesis, signal modeling, and natural language. $\mathrm{He}$ is a member of the Acoustical Society of Japan, the Institute of Electronics, Information and Communications Engineers, Japan, and the IEEE. He received the Invention Prize from the Institute of Invention, Japan, in 1991. 